\title{
ON MONOSPLINES OF LEAST DEVIATION $\left({ }^{1}\right)$
}

\author{
BY \\ R. S. JOHNSON
}

Introduction and statement of results. Let

$$
\xi_{1}<\xi_{2}<\cdots<\xi_{k}
$$

be $k$ given real numbers, and $n$ a non-negative integer. Let the function $S$ be defined in each of the intervals $\left(-\infty, \xi_{1}\right),\left[\xi_{1}, \xi_{2}\right), \cdots,\left[\xi_{k}, \infty\right)$ by a separate polynomial of degree not exceeding $n$, so that the composite function $S$ be continuous together with its first $n-1$ derivatives (no continuity requirement for $n=0) . S^{(n)}$ is a step-function with (possible) discontinuities at the points (1). A function $S$ of this kind is called a spline function [7] of class $(n, k)$, the points (1) being referred to as its knots.

It is convenient to introduce the notation

$$
x_{+}=\left\{\begin{array}{ll}
x, & x \geqq 0 \\
0, & x<0
\end{array},\right.
$$

in terms of which the most general spline function of class $(n, k)$ with knots (1) may be written as

$$
S(x)=P_{n}(x)+\sum_{\nu=1}^{k} \rho_{\nu}\left(x-\xi_{\nu}\right)_{+}^{n},
$$

where $P_{n}$ is a polynomial of degree at most $n$ and the $\rho_{\nu}$ are arbitrary numbers. Thus $S$ depends on $n+2 k+1$ arbitrary parameters.

Conventions. Throughout the remainder of this paper it is to be understood that $n \geqq 1, k \geqq 0$, whenever these symbols are used, unless the contrary is stated. We agree to take $(0)_{+}^{0}=1,(x)_{+}^{0}=0$ for $x<0$.

A monospline of class $(n, k)$ with knots (1) is a function $M$ of the form

$$
M(x)=x^{n}+S(x),
$$

where $S$ is a spline function of class $(n-1, k)$ with knots (1). Thus a monospline of class $(n, k)$ depends on $n+2 k$ parameters.

Among all polynomials of degree $n$ with leading coefficient one, the Tchebycheff polynomial $T_{n}[5$, p. 36] defined by

$$
T_{n}(x)=2^{-(n-1)} \cos (n \arccos x), \quad-1 \leqq x \leqq 1,
$$

Received by the editors February 27, 1959 and, in revised form, October 9, 1959.

(1) This paper is a dissertation prepared under the supervision of Professor I. J. Schoenberg and accepted by the Graduate School of the University of Pennsylvania in partial fulfillment of the requirements for the Ph.D. degree. 
deviates least from zero on the interval $[-1,1]$. The maximum absolute deviation is clearly $2^{-(n-1)}$, and it is achieved at the $n+1$ points $x=\cos (\pi j / n)$, $0 \leqq j \leqq n$, with alternating signs. We investigate here the existence, uniqueness, and properties of monosplines of class $(n, k)$ which deviate least from zero on $[-1,1]$. Letting $M_{n, k}^{*}$ denote any such monospline of class $(n, k)$ we have

$$
M_{n, 0}^{*}=T_{n},
$$

since a monospline of class $(n, 0)$ is a polynomial of degree $n$ with leading coefficient one.

It is shown in $\S 2$ that a monospline of class $(n, k)$ can have at most $n+2 k$ zeros. Thus a monospline of class $(n, k), n \geqq 3$, being proportional to the integral of a continuous monospline of class $(n-1, k)$, can have at most $n+2 k-1$ relative extrema. This limitation holds also for $n=1, n=2$. The main results obtained in this paper are contained in the following two theorems.

THEOREM 1. Given any set of numbers $\left\{e_{1}, \cdots, e_{n+2 k-1}\right\}$, such that

$$
\begin{array}{lll}
e_{n+2 k-1}<e_{n+2 k-2}, & e_{n+2 k-3}<e_{n+2 k-4}, \cdots, & n+2 k \geqq 2, \\
e_{n+2 k-2}>e_{n+2 k-3}, & e_{n+2 k-4}>e_{n+2 k-5}, \cdots, &
\end{array}
$$

there exists a monospline $M_{n, k}$ of class $(n, k)$ which has the $e_{\nu}$ as its relative extrema, in the given order. That is, for $n \geqq 2$ there is a sequence $x_{1}<x_{2}<\ldots$ $<x_{n+2 k-1}$ such that $M_{n, k}\left(x_{\nu}\right)=e_{\nu}, 1 \leqq \nu \leqq n+2 k-1$, and that the $e_{\nu}$ are the relative extrema of $M_{n, k}$. For $n=1$, there is a sequence $x_{1}<x_{2}<\cdots<x_{k}$ such that $M_{1, k}\left(x_{\nu}-\right)=e_{2 \nu-1}, M_{1, k}\left(x_{\nu}+\right)=e_{2 \nu}, 1 \leqq \nu \leqq k$, and that the $e_{\nu}$ are the relative extrema of $M_{1, k}$.

THEOREM 2. For each $(n, k)$ there exists a unique monospline $M_{n, k}^{*}$ of class $(n, k)$ which deviates least from zero on $[-1,1]$. For $n \geqq 2, M_{n, k}^{*}$ achieves its maximum absolute deviation, with alternating signs, at precisely $n+2 k+1$ points of $[-1,1]$, including both end-points, and this condition determines $M_{n, \mathrm{k}}^{*}$ uniquely.

It is to be noted that the family $S_{n-1, k}$ of $(n-1, k)$-splines is not solvent (for $k>0$ ) in the sense of Motzkin [4]. For, since $S_{n-1, k}$ is an $(n+2 k)$-parametric family of continuous functions (for $n>1$ ), any member $S$ of this family such that $S(-1+2 v /(n+2 k-1))=(-1)^{\nu}, \nu=0,1, \cdots, n+2 k-1$, would necessarily have at least $n+2 k-1$ zeros, whereas such a function $S$ can have at most $n+k-1$ zeros by Theorem 4 . Furthermore, the subfamilies $\delta_{n-1, k}^{N}$ of $(n-1, k)$-splines which are bounded in absolute value by $N$ in some fixed interval are not closed, as is easily seen by considering the sequence

$$
S_{n-1,2}^{(m)}(x)=\frac{m}{2(n-1)}\left[\left(x+\frac{1}{m}\right)_{+}^{n-1}-\left(x-\frac{1}{m}\right)_{+}^{n-1}\right], \quad m=2,3, \cdots .
$$


for any fixed integer $n>1$. Each $S_{n-1,2}^{(m)}$ is an element of $S_{n-1,2}$, and $\left|S_{n-1,2}^{(m)}(x)\right|$ $\leqq(3 / 2)^{n-2},-1 \leqq x \leqq 1$, but

$$
S(x)=\lim _{m \rightarrow \infty} S_{n-1,2}^{(m)}(x)=(x)_{+}^{n-2}
$$

is not an element of $\delta_{n-1,2}$ (but rather of $\delta_{n-2,2}$ ). Hence we may not appeal to the results of [4] in proving the above theorems. We are indebted to the referee for calling our attention to this reference.

The above theorems are proved in $\S 3$. For polynomials, $k=0$, Theorem 1 is a special case of a theorem of MacLane [3, pp. 100, 101]. A qualitative result of a similar nature is obtained by Kempner [2], where it is shown that the relative magnitudes of the maxima and the minima of a polynomial with a maximal number of extrema may be prescribed arbitrarily, subject to the necessary alternation of maxima and minima.

In $\S 1$ we construct the functions $M_{n, k}^{*}$ for $n=1,2,3$, and 4, using Theorem 2 for $n=3$ and 4 , and offer a short proof of the existence of a monospline of class $(n, 1)$ having $n+3$ absolutely equal extrema in $[-1,1]$. In $\S 2$ we discuss the zeros of spline functions and monosplines. In $\$ 4$ we investigate the magnitude of the absolute deviation from zero of $M_{n, \mathbf{k}}^{*}$ in $[-1,1]$.

1. Explicit formulas. We consider the problem of constructing the monospline $M_{n, k}^{*}$ of class $(n, k)$ which deviates least from zero in $[-1,1]$, for arbitrary $k$ and small values of $n$. When we speak of the scaled (Tchebycheff) polynomial $\tau_{n}$ appropriate to an interval $[a, b]$, we refer to the polynomial

$$
T_{n}(x)=\left(\frac{b-a}{2}\right)^{n} T_{n}\left[\frac{2 x-(b+a)}{b-a}\right], \quad a \leqq x \leqq b .
$$

The cases $n=1$ and $n=2$ are particularly simple, by the following reasoning. The interval $[-1,1]$ is subdivided into $r+1 \leqq k+1$ subintervals by the $r \leqq k$ knots of $M_{n, k}^{*}$ which lie in $[-1,1]$. In each of these subintervals $M_{n, k}^{*}$ cannot deviate less from zero than does the scaled polynomial $\tau_{n}$ appropriate to that interval. Hence it is clear that, if the $k$ knots be so chosen as to subdivide $[-1,1]$ into $k+1$ equal subintervals, and if the scaled polynomials $\tau_{n}$ appropriate to these intervals join smoothly enough at the knots that the composite function be a monospline, then this monospline is the function $M_{n, k}^{*}$, whose existence and uniqueness are thereby proved.

(a) For $n=1$ there are no continuity requirements, so the above conditions can surely be satisfied. The function $M_{1, k}^{*}$ is easily seen to be given by

$$
\begin{aligned}
& M_{1, k}^{*}(x)=\frac{1}{k+1} T_{1}[(k+1) x-k], \quad \frac{k-1}{k+1} \leqq x, \\
& M_{1, k}^{*}\left(x-\frac{2 j}{k+1}\right)=M_{1, k}^{*}(x), \quad \frac{k-1}{k+1} \leqq x<1, \quad 1 \leqq j \leqq k-1, \\
& M_{1, k}^{*}(-x)=-M_{1, k}^{*}(x), \quad \frac{k-1}{k+1}<x .
\end{aligned}
$$


(b) For $n=2$, the separate parabolic arcs of $M_{2, \mathrm{k}}^{*}$ must join continuously at the end-points of the subintervals determined by the knots $\xi_{j}$. Since $T_{2}(-1)=T_{2}(1)$, this requirement is fulfilled by the appropriately scaled polynomials $\tau_{2}$. Thus the function $M_{2, \mathbf{k}}^{*}$ is given by

$$
\begin{aligned}
& M_{2, k}^{*}(x)=\frac{1}{(k+1)^{2}} T_{2}[(k+1) x-k], \quad \frac{k-1}{k+1} \leqq x, \\
& M_{2, k}^{*}\left(x-\frac{2 j}{k+1}\right)=M_{2, k}^{*}(x), \quad \frac{k-1}{k+1} \leqq x<1, \quad 1 \leqq j \leqq k-1, \\
& M_{2, k}^{*}(-x)=M_{2, k}^{*}(x), \quad \frac{k-1}{k+1}<x .
\end{aligned}
$$

(c) The above device will not work for $n=3$, since $T_{3}(-1)=-T_{3}(1)$, and a direct construction seems difficult. However, Theorem 2 provides enough additional information about $M_{3, \mathbf{k}}^{*}$ to permit us to determine it rather easily. Instead, we shall verify that

$$
\begin{aligned}
& M_{3, k}^{*}(x)=\frac{1}{\left(\left(3^{1 / 2} / 2\right) k+1\right)^{3}} T_{3}\left\{\frac{3^{1 / 2}}{2}\left[\left(k+\frac{2}{3} 3^{1 / 2}\right) x-k\right]\right\}, \\
& \frac{k-1}{k+(2 / 3) 3^{1 / 2}} \leqq x, \\
& M_{3, k}^{*}\left(x-\frac{2 j}{k+(2 / 3) 3^{1 / 2}}\right)=M_{3, k}^{*}(x), \\
& \frac{k-1}{k+(2 / 3) 3^{1 / 2}} \leqq x<\frac{k+1}{k+(2 / 3) 3^{1 / 2}}, \quad 1 \leqq j \leqq k-1, \\
& M_{3, k}^{*}(-x)=-M_{3, k}^{*}(x), \quad \frac{k-1}{k+(2 / 3) 3^{1 / 2}}<x .
\end{aligned}
$$

First of all, noting that the absolutely equal extrema of $T_{3}(y)$ are located at $y= \pm 1$ and $y= \pm 1 / 2$, we see that $M_{3, k}^{*}(x)$ has absolutely equal extrema at the points

$$
x=\frac{k \pm(1 / 3) 3^{1 / 2}}{k+(2 / 3) 3^{1 / 2}} .
$$

Hence, $M_{3, k}^{*}$ has two absolutely equal extrema in each of the intervals

$$
\frac{k-2 j-1}{k+(2 / 3) 3^{1 / 2}}<x \leqq \frac{k-2 j+1}{k+(2 / 3) 3^{1 / 2}}, \quad 0 \leqq j \leqq k .
$$

and one more at each of the points \pm 1 , making $2 k+4$ in all. According to Theorem 2, if we can show that 


$$
M_{3, k}^{*(i)}\left(\frac{k-2 j-1}{k+(2 / 3) 3^{1 / 2}}-\right)=M_{3, k}^{*(i)}\left(\frac{k-2 j-1}{k+(2 / 3) 3^{1 / 2}}+\right), 0 \leqq j \leqq k-1, i=0,1,
$$

it will follow that $M_{3, k}^{*}$ is correctly defined by (10). Hence it is enough to show that

$$
T_{3}^{(i)}\left(-\frac{1}{2} 3^{1 / 2}\right)=T_{3}^{(i)}\left(\frac{1}{2} 3^{1 / 2}\right), \quad i=0,1,
$$

which is clearly the case since $T_{3}{ }^{\prime}$ is an even function and $T_{3}\left( \pm(1 / 2) 3^{1 / 2}\right)=0$.

(d) For $n=4$ we again appeal to Theorem 2 in verifying that $M_{4, k}^{*}$ is correctly defined by

$$
\begin{aligned}
& M_{4, k}^{*}(x)=\frac{1}{\left((1 / 2) 2^{1 / 2} k+1\right)^{4}} T_{4}\left\{\frac{2^{1 / 2}}{2}\left[\left(k+2^{1 / 2}\right) x-k\right]\right\}, \quad \frac{k-1}{k+2^{1 / 2}} \leqq x, \\
& M_{4, k}^{*}\left(x-\frac{2 j}{k+2^{1 / 2}}\right)=M_{4, k}^{*}(x), \quad \frac{k-1}{k+2^{1 / 2}} \leqq x<\frac{k+1}{k+2^{1 / 2}}, \\
& M_{4, k}^{*}(-x)=M_{4, k}^{*}(x), \quad \frac{k-1}{k+2^{1 / 2}}<x .
\end{aligned}
$$

Since the absolutely equal extrema of $T_{4}(y)$ are located at $y=0, y= \pm 2^{1 / 2} / 2$, and $y= \pm 1$, we see that $M_{4, k}^{*}$ has absolutely equal extrema at the points

$$
x=\frac{k}{k+2^{1 / 2}}, \quad x=\frac{k \pm 1}{k+2^{1 / 2}} .
$$

Hence, $M_{4, \mathbf{k}}^{*}$ has two absolutely equal extrema in each of the intervals

$$
\frac{k-2 j-1}{k+2^{1 / 2}}<x \leqq \frac{k-2 j+1}{k+2^{1 / 2}}, \quad 0 \leqq j \leqq k,
$$

and one more at each of the points $x= \pm 1, x=-(k+1) /\left(k+2^{1 / 2}\right)$, making $2 k+5$ in all. By Theorem 2 , if we can show that

$$
M_{4, k}^{*(i)}\left(\frac{k-2 j-1}{k+2^{1 / 2}}-\right)=M_{4, k}^{*(i)}\left(\frac{k-2 j-1}{k+2^{1 / 2}}+\right), \quad 0 \leqq j \leqq k-1, i=0,1,2,
$$

it will follow that $M_{\mathbf{4}, \mathbf{k}}^{*}$ is correctly defined by (11). Hence it is enough to show that

$$
T_{4}^{(i)}\left(-\frac{1}{2} 2^{1 / 2}\right)=T_{4}^{(i)}\left(\frac{1}{2} 2^{1 / 2}\right), \quad i=0,1,2 ;
$$

these equations are satisfied, since $T_{4}$ and $T_{4}^{(2)}$ are even functions, while $T_{4}^{\prime}\left( \pm(1 / 2) 2^{1 / 2}\right)=0$. 
It might be inferred from the foregoing that in all cases $M_{n, k}^{*}$ was made up of pieces of the polynomial $T_{n}$. In order for this to be so, it would have to be true that

$$
T_{n}^{(j)}(x-b)=T_{n}^{(j)}(x), \quad 0 \leqq j \leqq n-2,
$$

for some pair $(x, b)$ such that $-1 \leqq x-b \leqq 1,-1 \leqq x \leqq 1, b \neq 0$, and such a pair does not exist for $n \geqq 5$. To see this, we may use the well known result

$$
T_{n}(x)=\sum_{k=0}^{[n / 2]}(-1)^{k} \frac{n}{n-k}\left(\begin{array}{c}
n-k \\
k
\end{array}\right) 4^{-k} x^{n-2 k}
$$

to show that

$$
\begin{array}{ccc}
T_{n}^{(n-5)}(x)=\frac{n !}{5 !} x^{5}-\frac{n(n-2) !}{4 !} x^{3}+\frac{n(n-3) !}{32} x, & n \geqq 5, \\
T_{n}^{(n-3)}(x)=\frac{n !}{3 !} x^{3}-\frac{n(n-2) !}{4} x, & n \geqq 3, \\
T_{n}^{(n-2)}(x)=\frac{n !}{2 !} x^{2}-\frac{n(n-2) !}{4}, & n \geqq 2,
\end{array}
$$

By the third of these equations, in order that $T_{n}^{(n-2)}(x-b)=T_{n}^{(n-2)}(x)$ we must have $b(b-2 x)=0$. If $b=2 x$, the equations to be satisfied are $T_{n}^{(j)}(-x)$ $=T_{n}^{(j)}(x), 0 \leqq j \leqq n-2$. Since $T_{n}^{(n-3)}$ and $T_{n}^{(n-5)}$ are odd functions, we must have $T_{n}^{(n-3)}(x)=T_{n}^{(n-5)}(x)=0$. Now the nonzero roots of $T_{n}^{(n-3)}(x)=0$ are

$$
x= \pm\left(\frac{3}{2(n-1)}\right)^{1 / 2}
$$

while those of $T_{n}^{(n-5)}(x)=0$ are

$$
x= \pm\left(\frac{5 \pm\left(\frac{2 n-7}{n-2}\right)^{1 / 2}}{2(n-1)}\right)^{1 / 2},
$$

and there is no common solution. Hence $b=0$, and the desired condition cannot be satisfied.

It is of course possible to write down the equations which must be satisfied by $M_{n, k}^{*}$ as a consequence of Theorem 2, and these equations suffice in principle to determine the solution completely. They are very cumbersome, however, and no explicit formulas have been obtained for $n \geqq 5$.

It may be of interest to note that the existence of a monospline of class $(n, 1)$ having $n+3$ absolutely equal extrema in $[-1,1]$ can be shown more directly than via the argument of $\S 3$. Such a monospline must be the function $M_{n, 1}^{*}$, by Theorem 2 . 
In view of the uniqueness guaranteed by Theorem 2, such a function must have its single knot $\xi$ at the origin, and must be even or odd according as $n$ is even or odd. We consider first the case $n$ odd and $\geqq 3$, and let

$$
f_{n}(x)=\left\{\begin{aligned}
x^{n-1}, & x \geqq 0 \\
-x^{n-1}, & x<0 .
\end{aligned}\right.
$$

Let $Q_{n}$ be the Tchebycheffian approximation to $f_{n}$ on $[-1,1]$ by a polynomial of degree at most $n+1$. $Q_{n}$ is known to exist, be unique, and to be such that $f_{n}-Q_{n}$ has $n+3$ absolutely equal extrema on $[-1,1]$ (see [5, pp. 25-32]). Since $f_{n}$ is odd and $Q_{n}$ is unique, $Q_{n}$ must itself be odd, and so

$$
f_{n}(x)-Q_{n}(x)=\sum_{j=1}^{(n+1) / 2} \alpha_{2 j-1} x^{2 j-1}+x^{n-1}-2(-x)_{+}^{n-1} .
$$

We assert that $\alpha_{n} \neq 0$. For, if $\alpha_{n}=0$, then $f_{n}-Q_{n}$ is a spline function of class $(n-1,1)$, and as such can have at most $n$ zeros (see Theorem 4 ), while it is known to change sign at least $n+2$ times. Hence we may divide by $\alpha_{n}$, obtaining the function $M_{n, 1}$ defined by

$$
M_{n, 1}(x)=x^{n}+\frac{x^{n-1}-2(-x)_{+}^{n-1}}{\alpha_{n}}+\sum_{j=1}^{(n-1) / 2} \frac{\alpha_{2 j-1}}{\alpha_{n}} x^{2 j-1}
$$

having $n+3$ absolutely equal extrema in $[-1,1]$. It is easy to see that extrema must occur at both points $x= \pm 1$, and thus $M_{n, 1}=M_{n, 1}^{*}$. When $n$ is even, we approximate instead of $f_{n}$ the function $g_{n}$ defined by $g_{n}(x)=|x|^{n-1}$, and proceed exactly as above.

We note also that, with the aid of equations (8)-(11) and the above discussion, we can determine the Tchebycheffian approximation $C_{n}$ to the function $h_{n}$ defined by

$$
h_{n}(x)=x^{n-1}+(-1)^{n} 2(-x)_{+}^{n-1}, \quad n=2,3,4,
$$

(called $f_{n}$ or $g_{n}$ above according as $n$ was odd or even) on $[-1,1]$ by a polynomial of degree at most $n$. The explicit formulas are:

$$
\begin{array}{ll}
\text { (17) } h_{2}(x)=|x|, & C_{2}(x)=x^{2}+\frac{1}{8} \\
\text { (18) } h_{3}(x)=\left\{\begin{array}{ll}
x^{2}, x \geqq 0 \\
-x^{2}, & x<0
\end{array}, \quad C_{3}(x)=\left(\frac{3+2\left(3^{1 / 2}\right)}{9}\right) x^{3}+\left(\frac{4\left(3^{1 / 2}\right)-6}{3}\right) x\right. \\
\text { (19) } h_{4}(x)=|x|^{3}, \quad C_{4}(x)=\left(\frac{1+2^{1 / 2}}{4}\right) x^{4}+\left(2^{1 / 2}-1\right) x^{2}-\frac{1}{8}\left(2^{1 / 2}-1\right)^{3} .
\end{array}
$$

2. The zeros of spline functions and monosplines. Let $S$ be a spline function of class $(n, k), n \geqq 0$, with knots (1), and assume for the moment that 
there is no interior interval in which $S$ vanishes identically, i.e., if $\xi_{1}<x<\xi_{k}$ there is a deleted neighborhood $\hat{N}_{x}$ of $x$ such that $S(t) \neq 0$ for all $t$ in $\hat{N}_{x}$. Then, if $x \neq \xi_{\nu}, 1 \leqq \nu \leqq k, S$ has a zero of order $r \leqq n$ at $x$ if and only if

$$
S(x)=S^{\prime}(x)=\cdots=S^{(r-1)}(x)=0, \quad S^{(r)}(x) \neq 0 .
$$

The same definition will also be used when $x=\xi_{\nu}$ for some $1 \leqq \nu \leqq k$, provided that $r \leqq n-1$. However, the case $x=\xi_{\nu}, r=n$, requires special treatment. In this event clearly

$$
S(x)= \begin{cases}A\left(x-\xi_{\nu}\right)^{n}, & x<\xi_{\nu}, \\ B\left(x-\xi_{\nu}\right)^{n}, & x \geqq \xi_{\nu},\end{cases}
$$

in some neighborhood of the point $\xi_{v}$. We make the convention that

$$
\begin{aligned}
& \text { if } A B>0, \quad \xi_{\nu} \text { is a zero of order } n ; \\
& \text { if } A B \leqq 0, \quad \xi_{\nu} \text { is a zero of } \operatorname{order} n+1 .
\end{aligned}
$$

If there is a (maximal) interior interval $[a, b]$ in which $S$ vanishes identically, we assign to that interval a zero of whatever order the above definition ascribes to the "composite point" $\{a, b\}$ if the interval is considered as being shrunk to a point. Let $S_{n, k}$ denote an arbitrary spline function of class $(n, k)$, and let $Z\left(S_{n, k}\right)$ denote the number of zeros of $S_{n, k}$, counting multiplicities as above. Then

Theorem 3. $Z\left(S_{n, k}^{\prime}\right) \geqq Z\left(S_{n, k}\right)-1$, where $S_{n, k}^{\prime}(x) \equiv(d / d x) S_{n, k}(x)$.

Proof. In view of the above convention about maximal intervals of identical vanishing, it is enough to consider the case in which $S_{n, k}$ has only isolated zeros. If $S_{n, k}$ has a zero of order $r_{\nu} \geqq 1$ at each of the distinct points $x_{\nu}, 1 \leqq \nu$ $\leqq m$, then $S_{n, k}^{\prime}$ has by definition a zero of order $r_{\nu}-1$ at each $x_{\nu}$. If $n \geqq 2, S_{n, k}^{\prime}$ is continuous, and so has at least one zero in each of the $m-1$ intervals $\left(x_{\nu}, x_{\nu+1}\right), 1 \leqq \nu \leqq m-1$, by Rolle's theorem. If $n=1,\left|S_{1, k}\right|$ has a maximum in each interval $\left(x_{\nu}, x_{\nu+1}\right)$, say at $x=t_{\nu}$. (If there is a maximal subinterval throughout which $\left|S_{1, k}\right|$ assumes its constant maximum value, let $t_{\nu}$ be the left-hand endpoint of that subinterval.) Now $S_{1, t}^{\prime}$ is a spline function of class $(0, k)$, and $S_{1, k}^{\prime}\left(t_{\nu}-\right) \times S_{1, k}^{\prime}\left(t_{\nu}+\right) \leqq 0$, using again the convention about intervals of identical vanishing if necessary. Hence $S_{1, k}^{\prime}$ has a zero of order one at $t_{\nu}$, by definition. Thus in any case

$$
Z\left(S_{n, k}^{\prime}\right) \geqq \sum_{\nu=1}^{m}\left(r_{\nu}-1\right)+(m-1)=\sum_{\nu=1}^{m} r_{\nu}-1=Z\left(S_{n, k}\right)-1 .
$$

ThEOREM 4. $Z\left(S_{n, k}\right) \leqq n+k, n \geqq 0$.

Proof. By $n$-fold application of Theorem 3 we find that $Z\left(S_{n, k}\right) \leqq Z\left(S_{n, k}^{(n)}\right)$ $+n$. But $S_{n, k}^{(n)}$ is a spline function of class $(0, k)$, and as such can clearly have no more zeros than knots. Hence $Z\left(S_{n, k}^{(n)}\right) \leqq k, Z\left(S_{n, k}\right) \leqq n+k$. 
Thus we have $Z\left(S_{n, k}\right) \leqq Z\left(S_{n, \mathbf{k}}^{\prime}\right)+1 \leqq \cdots \leqq Z\left(S_{n, \mathbf{k}}^{(n)}\right)+n \leqq n+k$. Hence, if $Z\left(S_{n, k}\right)=n+k$, we have $Z\left(S_{n, k}^{(j)}\right)=n+k-j, 0 \leqq j \leqq n$.

Slightly different definitions will be employed in the case of monosplines $M_{n, k}$ of class $(n, k)$ with knots (1). Here there is of course no possibility of an interval of identical vanishing. The definition (20) of a zero of order $r$ at a point $x$ will still be used if $x$ is not a knot, or if $x$ is a knot $\xi_{\nu}$ and $r \leqq n-2$. If $x=\xi_{\nu}$ and

then clearly

$$
M_{n, k}\left(\xi_{\nu}\right)=M_{n, k}^{\prime}\left(\xi_{\nu}\right)=\cdots=M_{n, k}^{(n-2)}\left(\xi_{\nu}\right)=0,
$$

$$
M_{n, k}(x)=\left\{\begin{array}{ll}
\left(x-\xi_{\nu}\right)^{n}+A\left(x-\xi_{\nu}\right)^{n-1}, & x<\xi_{\nu} \\
\left(x-\xi_{\nu}\right)^{n}+B\left(x-\xi_{\nu}\right)^{n-1}, & x \geqq \xi_{\nu}
\end{array} \quad A \neq B,\right.
$$

in some neighborhood of the point $\xi_{v}$. We make the convention that

$$
\text { if } A B>0, \xi_{\nu} \text { is a zero of order } n-1 \text {; }
$$

if $A B<0, \xi_{\nu}$ is a zero of order $n$;

if $A B=0$ and $B-A>0, \xi_{\nu}$ is a zero of order $n$;

if $A B=0$ and $B-A<0, \xi_{p}$ is a zero of order $n+1$.

Then as above we have

Theorem 5. $Z\left(M_{n, \mathbf{k}}^{\prime}\right) \geqq Z\left(M_{n, k}\right)-1, n \geqq 2, M_{n, \mathbf{k}}^{\prime}(x) \equiv(d / d x) M_{n, k}(x)$.

Proof. If $M_{n, k}$ has a zero of order $r_{\nu} \geqq 1$ at each of the distinct points $x_{\nu}, 1 \leqq \nu \leqq m$, then $M_{n, k}^{\prime}$, being proportional to a monospline of class $(n-1, k)$, has by definition a zero of order $r_{\nu}-1$ at each $x_{\nu}$. If $n \geqq 3, M_{n, \mathbf{k}}^{\prime}$ is continuous, and so has at least one zero in each of the $m-1$ intervals $\left(x_{\nu}, x_{\nu+1}\right), 1 \leqq \nu \leqq m-1$, by Rolle's theorem. If $n=2,\left|M_{2, k}\right|$ has a maximum in each interval $\left(x_{\nu}, x_{\nu+1}\right)$, say at $x=t_{\nu}$. Now either $t_{\nu}$ is a continuity point of $M_{2, k}^{\prime}$, in which case $M_{2, k}^{\prime}\left(t_{\nu}\right)$ $=0$, or else $t_{v}$ is a knot of $M_{2, k}$ and

$$
M_{2, k}^{\prime}\left(t_{\nu}-\right) \times M_{2, k}^{\prime}\left(t_{\nu}+\right) \leqq 0 .
$$

But $(1 / 2) M_{2, k}^{\prime}$ is a monospline of class $(1, k)$, and so this inequality implies that $M_{2, \mathbf{k}}^{\prime}\left(t_{\nu}\right)=0$, by $(23)$ and $(24)$. Hence in any case $Z\left(M_{n, \mathbf{k}}^{\prime}\right) \geqq \sum_{\nu=1}^{m}\left(r_{\nu}-1\right)$ $+(m-1)=Z\left(M_{n, k}\right)-1$.

THEOREM 6. $Z\left(M_{n, k}\right) \leqq n+2 k$.

Proof. By $(n-1)$-fold application of Theorem 5 we find that $Z\left(M_{n, k}\right)$ $\leqq Z\left(M_{n, k}^{(n-1)}+(n-1)\right.$. But $M_{n, k}^{(n-1)}$ is proportional to a monospline of class $(1, k)$, and thus can clearly have at most $2 k+1$ zeros. Hence $Z\left(M_{n, k}\right) \leqq n+2 k$.

Thus we have

$$
Z\left(M_{n, k}\right) \leqq Z\left(M_{n, k}^{\prime}\right)+1 \leqq \cdots \leqq Z\left(M_{n, k}^{(n-1)}\right)+n-1 \leqq n+2 k .
$$

Hence, if $Z\left(M_{n, k}\right)=n+2 k$, we have $Z\left(M_{n, k}^{(j)}\right)=n+2 k-j, 0 \leqq j \leqq n-1$. 
Let $\mathfrak{T}_{n, k}$ be the set of all monosplines of class $(n, k)$ having $n+2 k$ zeros in $[0,1]$, and let

$$
\sigma_{n, k}=\sup \left\{\left|M_{n, k}(x)\right|: M_{n, k} \in \mathfrak{T}_{n, k}, 0 \leqq x \leqq 1\right\} .
$$

We conjecture that $\sigma_{n, k}=\sigma_{n, k+1}$, in which case $\sigma_{n, k}=1$, since clearly $\sigma_{n, 0}=1$. At any rate we can prove

LEMMA 1. $\sigma_{n, k} \leqq n !$.

Proof. Let $M_{n, k} \in \mathscr{T}_{n, k}$; then $(1 / n !) M_{n, k}^{(n-1)} \in \mathscr{T}_{1, k}$, by the remark following Theorem 6. Since an element of $\mathfrak{T}_{1, k}$ must be of the form $x-c, 0 \leqq c \leqq 1$, at every point $x$ of $[0,1]$, we see that $\sigma_{1, k}=1$. Let $a_{j}$ be any zero of $M_{n, k}^{(j)}, 0 \leqq j$ $\leqq n-2$. Now

$$
M_{n, k}(x)=n ! \int_{a_{0}}^{x} \int_{a_{1}}^{t_{n-1}} \cdots \int_{a_{n-2}}^{t_{2}} \frac{1}{n !} M_{n, k}^{(n-1)}\left(t_{1}\right) d t_{1} d t_{2} \cdots d t_{n-1},
$$

and so

$$
\begin{aligned}
\left|M_{n, k}(x)\right| & \leqq n !\left|\int_{a_{0}}^{x} \int_{a_{1}}^{t_{n-1}} \cdots \int_{a_{n-2}}^{t_{2}}\right| \frac{1}{n !} M_{n, k}^{(n-1)}\left(t_{1}\right)\left|d t_{1} d t_{2} \cdots d t_{n-1}\right| \\
& \leqq n ! \int_{0}^{1} \int_{0}^{1} \cdots \int_{0}^{1} \sigma_{1, k} d t_{1} \cdots d t_{n-1}=n ! .
\end{aligned}
$$

Hence $\sup \left\{\left|M_{n, k}(x)\right|: M_{n, k} \in \Re_{n, k}, 0 \leqq x \leqq 1\right\}=\sigma_{n, k} \leqq n$ !.

We shall also require the following

Lemma 2. For any monospline $M_{n, k}$ of class $(n, k)$,

$$
\int_{0}^{1}\left|M_{n, k}(t)\right| d t \geqq 4(4 k+4)^{-(n+1)} .
$$

Proof. $M_{n, k}$ has at most $k$ knots in $[0,1]$, and hence there is at least one subinterval $[a, b] \subset[0,1]$ of length not less than $(k+1)^{-1}$ in which $M_{n, k}$ is a polynomial $P_{n}$ of degree $n$ with leading coefficient one. Thus

$$
\begin{aligned}
& \int_{0}^{1}\left|M_{n, k}(t)\right| d t \\
& \geqq \int_{a}^{b}\left|P_{n}(t)\right| d t=\frac{1}{2}(b-a) \int_{a}^{b}\left|P_{n}\left[\frac{1}{2}(b-a) x+\frac{1}{2}(b+a)\right]\right| d x \\
& =\left[\frac{1}{2}(b-a)\right]^{n+1} \int_{-1}^{1}\left|\frac{P_{n}\left[\left(2^{-1}\right)(b-a) x+\left(2^{-1}\right)(b+a)\right]}{\left[\left(2^{-1}\right)(b-a)\right]^{n}}\right| d x \\
& \geqq(2 k+2)^{-(n+1)} \inf _{P \in \Pi_{n}} \int_{-1}^{1}|P(x)| d x=(2 k+2)^{-(n+1)} \lambda_{n},
\end{aligned}
$$


where $\Pi_{n}$ denotes the class of all polynomials of degree $n$ with leading coefficient one. But it is known that $\lambda_{n}>0$, and in fact

$$
\lambda_{n}=\int_{-1}^{1}\left|U_{n}(x)\right| d x=2^{-(n-1)}
$$

where

$$
U_{n}(x)=\frac{1}{2^{n}} \frac{\sin [(n+1) \arccos x]}{\left(1-x^{2}\right)^{1 / 2}}, \quad-1 \leqq x \leqq 1,
$$

the modified Tchebycheff polynomial of degree $n$ [5, pp. 302-309].

3. Proofs of Theorems 1 and 2. In our proof of Theorem 1 we shall make use of a theorem of Schoenberg [8] which we state as

THEOREM 7. Given any sequence of numbers $x_{1}<x_{2}<\cdots<x_{n}+2 k$, there exists a unique monospline $M_{n, k}$ of class $(n, k)$ such that $M_{n, k}\left(x_{\nu}\right)=0,1 \leqq \nu$ $\leqq n+2 k . M_{n, k}$ depends continuously on the sequence $\left\{x_{\nu}\right\}$.

We remark that the knots $\left\{\xi_{\nu}\right\}$ of this monospline $M_{n, k}$ are related to its zeros $\left\{x_{\nu}\right\}$ by

$$
\begin{array}{ll}
x_{2 \nu}<\xi_{\nu}<x_{n+2 \nu-1}, & n>1 \\
x_{2 \nu}=\xi_{\nu}, & n=1
\end{array}
$$$$
1 \leqq \nu \leqq k
$$

The result for $n=1$ may be verified directly; in fact, the monospline $M_{1, k}$ is given by

$$
M_{1, k}(t)=\left(t-x_{1}\right)-\sum_{\nu=1}^{k}\left(x_{2 v+1}-x_{2 v-1}\right)\left(t-x_{2 v}\right)_{+}^{0},
$$

(see (23) and (24)). For $n>1$, we write

$$
M_{n, k}(t)=t^{n}+P_{n-1}(t)+\sum_{j=1}^{k} \rho_{j}\left(t-\xi_{j}\right)_{+}^{n-1} .
$$

Then if $\xi_{\nu} \geqq x_{n+2 v-1}$ the function $M$ defined by

$$
M(t)=t^{n}+P_{n-1}(t)+\sum_{j=1}^{\nu-1} \rho_{j}\left(t-\xi_{j}\right)_{+}^{n-1}
$$

has at least $n+2 \nu-1$ zeros, and is evidently a monospline of class $(n, \nu-1)$, contradicting Theorem 6 . Similarly, if $\xi_{\nu} \leqq x_{2 \nu}$ the function $M$ defined by

$$
M(t)=t^{n}+P_{n-1}(t)+\sum_{j=1}^{\nu} \rho_{j}\left(t-\xi_{j}\right)^{n-1}+\sum_{j=\nu+1}^{k} \rho_{j}\left(t-\xi_{j}\right)_{+}^{n-1}
$$

has at least $n+2(k-\nu)+1$ zeros, and is evidently a monospline of class $(n, k-\nu)$, again contradicting Theorem 6 . 
We also notice that, if $k=1$, (32) represents the necessary and sufficient condition that arbitrary values can be interpolated at any $n+1$ of the $n+2$ points $x_{\nu}$ by a spline function $S$ of class $(n, 1)$ with knot $\xi_{1}$ (see [7, pp. 256257]).

Before turning to the proof of Theorem 1, we dispose of the special case $n=1$ of that theorem. Here the situation is somewhat different than for larger values of $n$, in that the extrema $e_{2 \nu-1}, 1 \leqq \nu \leqq k$, are not actually assumed, i.e. they are suprema rather than maxima. We observe that the function $M_{1, k}$ defined by

$$
M_{1, k}(t)=t-\sum_{j=1}^{k}\left(e_{2 j-1}-e_{2 j}\right)\left[t-e_{2 j-1}-\sum_{i=1}^{j-1}\left(e_{2 i-1}-e_{2 i}\right)\right]_{+}^{0}
$$

is a monospline of class $(1, k)$ with the properties:

(i) $M_{1, k}$ is monotone increasing in each of the intervals

$$
\begin{array}{ll}
{\left[e_{2 \nu-1}+\sum_{j=1}^{\nu-1}\left(e_{2 j-1}-e_{2 j}\right), e_{2 v+1}+\sum_{j=1}^{\nu}\left(e_{2 j-1}-e_{2 j}\right)\right),} & 1 \leqq \nu \leqq k-1 \\
\left(-\infty, e_{1}\right),\left[e_{2 k-1}+\sum_{j=1}^{k-1}\left(e_{2 j-1}-e_{2 j}\right), \infty\right) ; &
\end{array}
$$

(ii) $M_{1, k}\left\{\left[e_{2 v-1}+\sum_{j=1}^{v-1}\left(e_{2 j-1}-e_{2 j}\right)\right]-\right\}=e_{2 v-1}$, $1 \leqq \nu \leqq k ;$

(iii) $M_{1, k}\left\{\left[e_{2 \nu-1}+\sum_{j=1}^{\nu-1}\left(e_{2 j-1}-e_{2 j}\right)\right]+\right\}=e_{2 \nu}$, $1 \leqq \nu \leqq k$.

Hence $M_{1, k}$ is a function of the type whose existence is asserted by Theorem 1 for $n=1$; in the sequel we consider only $n \geqq 2$, and prove the following

THEOREM 8. Suppose $N \geqq 3$, let $x=\left(x_{2}, \cdots, x_{N-1}\right)$ be an $(N-2)$-tuple of real numbers with $x_{1}=0<x_{2}<\cdots<x_{N-1}<1=x_{N}$, let $t$ be a real number, and let $f$ be a real-valued function of $t$ and $x$ satisfying the following conditions:

(i) $f(t, x)$ is continuous in $t$ for $0 \leqq t \leqq 1$, except possibly at some or all of the points $t=x_{j}$;

(ii) $|f(t, x)| \leqq h$ for $x$ as above, $0 \leqq t \leqq 1$;

(iii) $G(t, x)=\int_{0}^{t}|f(s, x)| d s$ is continuous in $x$ for $x$ as above;

(iv) $G(1, x) \geqq g>0$ for all $x$ as above;

(v) $f\left(x_{j}, x\right)=0$ for each $1 \leqq j \leqq N$ such that $f(t, x)$ is continuous at $t=x_{j}$, while $f(t, x) \neq 0$ for all other $0 \leqq t \leqq 1$;

(vi) $f(t, x)$ changes sign at each point $t=x_{j}, 1 \leqq j \leqq N$; for definiteness we suppose that $f(t, x)<0$ for $x_{N-1}<t<1$, so that $\operatorname{sgn}[f(t, x)]=(-1)^{N-i-1}, x_{j-1}<t$ $<x_{j}, 2 \leqq j \leqq N$. 
Let $F(t, x)=\int_{0}^{t} f(s, x) d s$, and let $\beta_{1}, \beta_{2}, \cdots, \beta_{N}$ be a sequence of real numbers such that $\beta_{1}=0$,

$$
\begin{aligned}
\beta_{N}<\beta_{N-1}, & \beta_{N-2}<\beta_{N-3}, \cdots, \\
\beta_{N-1}>\beta_{N-2}, & \beta_{N-3}>\beta_{N-4}, \cdots,
\end{aligned}
$$

so that $\operatorname{sgn}\left[\beta_{j}-\beta_{j-1}\right]=(-1)^{N-j-1}$. Then there exists $a$ vector $x$ and a number $\lambda>0$ such that

$$
F\left(x_{j}, x\right)=\lambda \beta_{j}, \quad 1 \leqq j \leqq N .
$$

Before proving Theorem 8 , we show that it implies Theorem $1, n \geqq 2$. In the above notation, let $N=n+2 k-1$ and let $f / n$ be the monospline of class $(n-1, k)$ which has a simple zero at each of the points $x_{j}$. Such a monospline exists uniquely, and depends continuously on the vector $x$, by Theorem 7 . Conditions (i), (v), and (vi) are clearly satisfied: $f$ is indeed negative in $\left(x_{N-1}, 1\right)$, since otherwise, being positive for $t$ sufficiently large, it would have a zero of even order at $t=1$. Conditions (ii) and (iv) are also fulfilled, according to Lemmas 1 and 2. Condition (iii) is surely met if $n \geqq 3$, since then $f$ is itself continuous in $t$ and $x$. If $n=2, f / 2$ is as given by (33) with $x_{1}=0$, $x_{2 k+1}=1$, and we find that

$$
\begin{array}{r}
G(t, x)=\sum_{v=1}^{k}\left\{\left[\left(t \cap x_{2 j}\right)-x_{2 j-1}\right]^{2}-\left[\left(t \cap x_{2 j-1}\right)-x_{2 j-1}\right]^{2}\right. \\
\left.+\left[x_{2 j+1}-\left(t \cap x_{2 j}\right)\right]^{2}-\left[x_{2 j+1}-\left(t \cap x_{2 j+1}\right)\right]^{2}\right\},
\end{array}
$$

where $a \cap b=\min \{a, b\}$. Hence $G$ is continuous in $x$, and the conditions of Theorem 8 are all satisfied. Let $\beta_{j}=e_{j}-e_{1}, 1 \leqq j \leqq n+2 k-1$. Then by Theorem 8 there exists a vector $x$ and a $\lambda>0$ such that (35) holds. $F$ is surely a monospline of class $(n, k)$, and hence is of the form

$$
F(t, x)=t^{n}+S_{n-1, k}(t)
$$

for some spline function $S_{n-1, k}$ of class $(n-1, k)$. But then the function $M_{n, k}$ defined by

$$
M_{n, k}(t)=t^{n}+\frac{1}{\lambda} S_{n-1, k}\left(\lambda^{1 / n} t\right)+e_{1}
$$

is a monospline of the type whose existence is asserted by Theorem 1 .

The cases $n \geqq 2, n+2 k-1=1$ or 2 , i.e. $k=0, n=2$ or 3 , are not covered by Theorem 8 . However, we may take for $F$ the functions defined by

$$
F(t, x)= \begin{cases}t^{2}, & n=2, k=0 \\ 2^{-1}\left(2 t^{3}-3 t^{2}\right), & n=3, k=0\end{cases}
$$

and the above argument still applies. 
We turn now to the proof of Theorem 8 , for which we shall require two additional lemmas. Let $\Phi$ denote the family of all real, continuous, strictly increasing functions $\phi$ defined on $[0,1]$, such that $\phi(0)=0$. For $\phi, \psi$ in $\Phi$, define $A(\phi, \psi)=\max \{|\phi(z)-\psi(z)|: 0 \leqq z \leqq 1\}$; then clearly $(\Phi, A)$ is a metric space. Let $\gamma_{2}, \gamma_{3}, \cdots, \gamma_{N}$ be a sequence of positive numbers.

Lemma 3. For every $\phi \in \Phi$ there is a unique subdivision $0<z_{2}<\cdots<z_{N-1}$ $<1$ of the interval $[0,1]$ such that

$$
\frac{\phi\left(z_{2}\right)}{\gamma_{2}}=\frac{\phi\left(z_{3}\right)-\phi\left(z_{2}\right)}{\gamma_{3}}=\cdots=\frac{\phi(1)-\phi\left(z_{N-1}\right)}{\gamma_{N}} .
$$

Proof. The existence of such a subdivision, but not its uniqueness, is a consequence of the $n$-lattice theorem [6]. We give a direct proof in this simple case. For any vector $z=\left(z_{2}, \cdots, z_{N-1}\right)$ which satisfies $(37)$ it is true that

$$
\frac{\phi\left(z_{2}\right)}{\gamma_{2}}=\frac{\phi\left(z_{3}\right)}{\gamma_{2}+\gamma_{3}}=\cdots=\frac{\phi(1)}{\sum_{j=1}^{N} \gamma_{j}} .
$$

Hence, $t=z_{\nu}$ is the unique solution of the equation

$$
t=\phi^{-1}\left\{\frac{\sum_{i=2}^{\nu} \gamma_{i}}{\sum_{i=2}^{N} \gamma_{i}} \phi(1)\right\}, \quad 2 \leqq \nu \leqq N-1 .
$$

For fixed $\gamma_{i}$ and $\phi$ as above, let $z^{\phi}=\left(z_{2}^{\phi}, \cdots, z_{N-1}^{\phi}\right)$ be the corresponding subdivision of $[0,1]$, and let $z_{1}^{\phi}=0, z_{N}^{\phi}=1$.

LEMMA 4. $z^{\phi}$ is a continuous function of $\phi$.

Proof. By (37) and (38) we have

$$
\frac{\phi\left(z_{j}^{\phi}\right)}{\phi(1)}=\frac{\sum_{i=2}^{i} \gamma_{i}}{\sum_{i=2}^{N} \gamma_{i}}=\lambda_{j}, \quad 1 \leqq j \leqq N .
$$

Suppose $A\left(\phi_{\nu}, \phi\right) \rightarrow 0$, and that for each positive integer $\nu$ and each $j$ we have also

$$
\frac{\phi_{\nu}\left(z_{j}^{\phi_{\nu}}\right)}{\phi_{\nu}(1)}=\lambda_{j}, \quad 1 \leqq j \leqq N .
$$

Given $0<\epsilon<1 / 2$, suppose that $A\left(\phi_{\nu}, \phi\right)<\epsilon$ for $\nu>\nu_{\epsilon}$. Then for such values of $\nu$ we have 


$$
(1-\epsilon) \phi\left(z_{j}^{\phi}\right)<\phi_{\nu}\left(z_{j}^{\phi^{\nu}}\right)<(1+\epsilon) \phi\left(z_{j}^{\phi}\right) \text {. }
$$

Suppose it has been established that $\left|z_{j}^{\phi}-z_{j}^{\phi}\right|<\epsilon$ for $j<\mu$ and $\nu>\nu_{\mu} \geqq \nu_{\epsilon}$. If $\mu=N$ the proof is complete by the definition of $z_{N}^{\phi}$. If $2 \leqq \mu<N$ there are two cases to consider.

(i) $z_{\mu}^{\phi} \leqq z_{\mu}^{\phi}$. Then

$$
\begin{gathered}
\phi_{\nu}\left(z_{\mu}^{\phi_{\nu}}\right)<(1+\epsilon)\left\{\phi\left(z_{\mu}^{\phi_{\nu}}\right)-\left[\phi\left(z_{\mu}^{\phi_{\nu}}\right)-\phi\left(z_{\mu}^{\phi}\right)\right]\right\} \\
0 \leqq \phi\left(z_{\mu}^{\phi_{\nu}}\right)-\phi\left(z_{\mu}^{\phi^{\prime}}\right)<\phi\left(z_{\mu}^{\phi_{\nu}}\right)-\phi_{\nu}\left(z_{\mu}^{\phi_{\nu}}\right)+\frac{\epsilon}{1+\epsilon} \phi_{\nu}\left(z_{\mu}^{\phi_{\nu}}\right)<\epsilon[1+\epsilon+\phi(1)] .
\end{gathered}
$$

(ii) $z_{\mu}^{\phi_{\nu}} \leqq z_{\mu}^{\phi}$. Then

$$
\begin{gathered}
(1-\epsilon)\left\{\phi\left(z_{\mu}^{\phi_{\nu}}\right)+\left[\phi\left(z_{\mu}^{\phi}\right)-\phi\left(z_{\mu}^{\phi_{\nu}}\right)\right]\right\}<\phi_{\nu}\left(z_{\mu}^{\phi_{\nu}}\right), \\
0 \leqq \phi\left(z_{\mu}^{\phi}\right)-\phi\left(z_{\mu}^{\phi_{\nu}}\right)<\phi_{\nu}\left(z_{\mu}^{\phi_{\nu}}\right)-\phi\left(z_{\mu}^{\phi_{\nu}}\right)+\frac{\epsilon}{1-\epsilon} \phi\left(z_{\mu}^{\phi_{\nu}}\right)<\epsilon[1+2 \phi(1)] .
\end{gathered}
$$

Hence in either case $\phi\left(z_{\mu}^{\phi_{\nu}}\right) \rightarrow \phi\left(z_{\mu}^{\phi}\right)$ as $\nu \rightarrow \infty$, and so $z_{\mu}^{\phi_{\nu}} \rightarrow z_{\mu}^{\phi}$ since $\phi$ is continuous and strictly increasing. Thus there is a $\nu_{\mu+1} \geqq \nu_{\mu}$ such that $\left|z_{j}^{\phi}-z_{j}^{\phi_{\nu}}\right|<\epsilon$ for $j<\mu+1$ and $\nu>\nu_{\mu+1} \geqq \nu_{\epsilon}$, and the truth of the lemma follows by induction.

Let $x$ and $f$ be as in the statement of Theorem 8 , and let $\left(\gamma_{2}, \cdots, \gamma_{N}\right)$ be a fixed sequence of positive numbers. Surely the function $G$ defined in condition (iii) is an element of $(\Phi, A)$; hence by Lemma 3 there exists a vector $z^{x}=\left(z_{2}^{x}, \cdots, z_{N-1}^{x}\right)$ such that $0<z_{2}^{x}<\cdots<z_{N-1}^{x}<1$, and such that

$$
\frac{G\left(z_{2}^{x}, x\right)}{\gamma_{2}}=\frac{G\left(z_{3}^{x}, x\right)-G\left(\underset{x}{z_{2}}, x\right)}{\gamma_{3}}=\cdots=\frac{G(1, x)-G\left(z_{N-1}^{x}, x\right)}{\gamma_{N}} .
$$

By Lemma 4 the mapping $T:\left(x_{2}, \cdots, x_{N-1}\right) \rightarrow\left(z_{2}^{x}, \cdots, z_{N-1}^{x}\right)$ is continuous on the open set $\left\{x: 0<x_{2}<\cdots<x_{N-1}<1\right\}$, since $z^{x}$ is a continuous function of $G$ and $G$ is continuous in $x$. The above equations may be rewritten as

$$
\int_{z_{i-1}^{x}}^{z_{j}^{x}}|f(t, x)| d t=\frac{\gamma_{j} \int_{0}^{1}|f(t, x)| d t}{\sum_{i=2}^{N} \gamma_{i}}, \quad 2 \leqq j \leqq N,
$$

so that

$$
\left(z_{j}^{x}-z_{j-1}^{x}\right) \sup \left\{|f(t, x)|: z_{j-1}^{x} \leqq t \leqq z_{j}^{x}\right\} \geqq \frac{\gamma_{j} \int_{0}^{1}|f(t, x)| d t}{\sum_{i=2}^{N} \gamma_{i}},
$$


by the first mean-value theorem, and

$$
\left(z_{j}^{x}-z_{j-1}^{x}\right) \geqq \frac{g \gamma_{j}}{h \sum_{i=2}^{N} \gamma_{i}}
$$

by (ii) and (iv) of Theorem 8 . Choose any positive $\epsilon$ less than

$$
\min \left\{\frac{1}{N}, \frac{g \gamma_{2}}{h \sum_{i=2}^{N} \gamma_{i}}, \cdots, \frac{g \gamma_{N}}{h \sum_{i=2}^{N}}\right\}
$$

Then we have shown that

$$
z_{j}^{x}-z_{j-1}^{x} \geqq \epsilon>0, \quad 2 \leqq j \leqq N .
$$

But this implies that the function $T$ above maps the closed $(N-2)$-simplex

$$
\left\{x: \epsilon \leqq x_{2} \leqq x_{3}-\epsilon \leqq \cdots \leqq x_{N-1}-(N-3) \epsilon \leqq 1-(N-2) \epsilon\right\}
$$

continuously into itself, and so the Brouwer Fixed Point Theorem (see [9, pp. 242-245]) guarantees the existence of at least one vector $x$ for which $\boldsymbol{z}^{x}=x$.

We can now complete the proof of Theorem 8. For, since $\left|\beta_{j}-\beta_{j+1}\right|>0$, $1 \leqq j \leqq N-1$, there exists by the above discussion a vector $x$ for which

$$
\frac{G\left(x_{2}, x\right)}{\left|\beta_{1}-\beta_{2}\right|}=\frac{G\left(x_{3}, x\right)-G\left(x_{2}, x\right)}{\left|\beta_{2}-\beta_{3}\right|}=\cdots=\frac{G(1, x)-G\left(x_{N-1}, x\right)}{\left|\beta_{N-1}-\beta_{N}\right|} .
$$

But since $f(t, x)$ is of constant sign in each interval $\left(x_{j-1}, x_{j}\right)$ it follows from (vi) that

$$
\begin{aligned}
G\left(x_{j}, x\right)-G\left(x_{j-1}, x\right) & =\int_{x_{j-1}}^{x_{j}}|f(t, x)| d t=(-1)^{N-j-1} \int_{x_{j-1}}^{x_{j}} f(t, x) d t \\
& =(-1)^{N-j-1}\left[F\left(x_{j}, x\right)-F\left(x_{j-1}, x\right)\right]
\end{aligned}
$$

$2 \leqq j \leqq N$, and hence (46) may be rewritten as

$$
\begin{aligned}
\frac{F\left(x_{2}, x\right)}{\beta_{2}} & =\frac{F\left(x_{3}, x\right)-F\left(x_{2}, x\right)}{\beta_{3}-\beta_{2}}=\cdots=\frac{F(1, x)-F\left(x_{N-1}, x\right)}{\beta_{N}-\beta_{N-1}} \\
& =\lambda>0,
\end{aligned}
$$

so that (35) holds and Theorem 8 is proved.

We base our proof of Theorem 2 on Theorem 1 and the following

THEOREM 9. If $n \geqq 2$, and if there exists a monospline $M_{n, k}$ of class $(n, k)$ and $a$ sequence of points $-1=x_{0}<x_{1}<\cdots<x_{n+2 k}=1$ such that $M_{n, k}$ has $a$ 
relative extremum at each point $x_{\nu}$ with $M_{n, k}\left(x_{\nu}\right)=(-1)^{\nu} M_{n, k}\left(x_{0}\right), 0 \leq \nu \leq n+2 k$, then $M_{n, k}=M_{n, k}^{*}$, so that the latter exists uniquely.

Proof. The unique $M_{2, k}$ with extrema in $\left[x_{0}, x_{2+2 k}\right]$ at $\left\{x_{v}\right\}_{0}^{2+2 k}$ is given by

$$
\begin{aligned}
M_{2, k}(x)= & M_{2, k}\left(x_{0}\right)-\left(x_{1}-x_{0}\right)^{2}+\left(x-x_{1}\right)^{2} \\
& -2 \sum_{\nu=1}^{k}\left(x_{2 \nu+1}-x_{2 \nu-1}\right)\left(x-x_{2 \nu}\right)_{+},
\end{aligned}
$$

as may easily be verified. The requirements $x_{0}=-1, M_{2, k}\left(x_{1}\right)=-M_{2, k}\left(x_{0}\right)$ imply that $2 M_{2, k}(-1)=\left(x_{1}+1\right)^{2}$, and by induction we find $\left(x_{j}-x_{j-1}\right)^{2}$ $=\left(x_{1}+1\right)^{2}, 1 \leqq j \leqq 2 k+2$. Hence the $x_{\nu}$ 's are equally spaced, and $M_{2, k}=M_{2, k}^{*}$ as given in (9) of $\S 1$. Thus we need consider only $n \geqq 3$.

Let $M_{n, k}$ be a monospline of class $(n, k), n \geqq 3$, let $-1=x_{0}<x_{1}<\cdots$ $<x_{n+2 k}=1$ be a sequence of points such that $M_{n, k}\left(x_{v}\right)=(-1)^{n-v} e_{0}$ for some $e_{0}>0$, with $\left|M_{n, k}(x)\right|<e_{0}$ for $x \neq x_{v}$, and let $N_{n, k}$ be any other monospline of class $(n, k)$ such that $\left|N_{n, k}(x)\right| \leqq e_{0},-1 \leqq x \leqq 1$. Assume that $M_{n, k} \neq N_{n, k}$; then $\Delta=M_{n, k}-N_{n, k}$ is a spline function of class $(n-1, r)$ for some $0 \leqq r \leqq 2 k$. Denote the interval $[-1,1]$ by $J$, and let $I$ be any maximal open subinterval of $J$ in which $\Delta$ vanishes identically. It is clear that an end-point of $I$ must be either a knot of $M_{n, k}$ or a knot of $M_{n, k}$ or one of the points $x= \pm 1$. Suppose that $I$ contains $\nu$ points which are knots of either $M_{n, k}$ or $N_{n, k}$, and note that they must in fact be common to both functions $M_{n, k}$ and $N_{n, k}$. Now $M_{n, k}$ is a monospline of class $(n, \nu)$ in $\bar{I}$, and $M_{n, k}^{\prime}$ is continuous. Hence $M_{n, k}^{\prime}$ must vanish at each extremum of $M_{n, k}$ in $\bar{I}$, unless one of the points $x= \pm 1$ is an end-point of $I$. Thus $M_{n, k}$ has at most $n+2 \nu-1$ extrema in $\bar{I}$, by Theorem 6 , unless one of the points $x= \pm 1$ is an end-point of $I$, in which case $M_{n, k}$ has at most $n+2 \nu$ extrema in $\bar{I}$.

Suppose there are exactly $p$ such intervals $I_{j}$, with $\nu_{j}$ knots common to both $M_{n, k}$ and $N_{n, k}$ in $I_{j}$. Then $\Delta$ has exactly $n p-p^{\prime}$ zeros in $\bigcup_{1}^{p} \bar{I}_{j}$, where $p^{\prime}=(p-2, p-1, p)$ according as (both, one, neither) of the points $x= \pm 1$ are found among the end-points of the intervals $I_{j}$. We wish to count the number of zeros of $\Delta$ in $K=J-\cup_{1}^{p} \bar{I}_{j}$. In doing this, according to the prescription given in $\S 2$, we must collapse each interval $I_{j}$ to a point. But then $\Delta$ is actually a spline function of class $\left(n-1,2 k-p^{\prime}-2 \sum_{1}^{p} \nu_{j}\right)$, since $2 \nu_{j}+1$ of the original $2 k$ knots are lost when each interval $I_{j}$ is collapsed to a point, unless one of the points $x= \pm 1$ is an end-point of $I_{j}$, when only $2 \nu_{j}$ knots are lost.

For each $j$, let $I_{j}^{\prime}$ be a relatively open subinterval of $J$ which contains $\bar{I}_{j}$ but which contains no zeros or knots of $\Delta$ or extrema of $M_{n, k}$ which are not already contained in $\bar{I}_{j}$. Then $M_{n, k}$ has at least $n+2 k+1-n p+p^{\prime}-2 \sum_{1}^{p} \nu_{j}$ extrema in $K^{\prime}=J-U_{1}^{p} I_{j}^{\prime}$, according to the above discussion, and $\Delta$ has only isolated zeros in $K^{\prime}$, and the number of zeros of $\Delta$ is the sum of those to be found in $K^{\prime}$ and the $n p-p^{\prime}$ already found in $\bigcup_{1}^{p} \bar{I}_{j}$. We remark that, by Theorem $4, \quad n p-p^{\prime} \leqq Z(\Delta) \leqq n+2 k-1-p^{\prime}-2 \sum_{1}^{p} \nu_{j} \leqq n+2 k-1-p^{\prime}$, and 
hence $p \leqq[(n+2 k-1) / n]$. Note also that $K^{\prime}$ is the disjoint union of $p^{\prime}+1$ closed subintervals of $J$.

To complete the proof of Theorem 2 we require two lemmas.

Lemma 5. If the closed interval $K_{j} \subset K^{\prime}$ contains $m$ of the points $x_{v}$, then $\Delta$ has at least $m-1$ zeros in $K_{j}$.

Proof. Since we consider only $n \geqq 3, M_{n, k}, N_{n, k}$, and $\Delta$ all have continuous first derivatives. Consider the subintervals $\left[x_{\nu_{1}}, x_{\nu_{2}}\right), \quad\left[x_{\nu_{2}}, x_{\nu_{3}}\right), \cdots$, $\left[x_{v_{m-1}}, x_{\nu_{m}}\right]$ of $K_{j}$, noting that $x_{v_{j}}$ is a maximum (minimum) of $M_{n, k}$ according as $n-\nu_{j}$ is even (odd). Suppose it has been established that, for some $2 \leqq j \leqq m$, either

(i) $\Delta$ has at least $j-1$ zeros on $\left[x_{\nu_{1}}, x_{\nu_{j-1}}\right]$, or

(ii) $\Delta$ has at least $j-2$ zeros on $\left[x_{v_{1}}, x_{v_{j-1}}\right.$ ), where (i) and (ii) are not necessarily exclusive. One of these conditions is surely met if $j=2$. Then, in case (i), $\Delta$ has at least $(j+1)-2$ zeros on $\left[x_{v_{1}}, x_{v_{j}}\right)$. In case (ii), either

(a) $\Delta\left(x_{v_{j-1}}\right)=0$, and $\Delta^{\prime}\left(x_{v_{j-1}}\right)=0$ if $j \geqq 3$, or

(b) $\Delta\left(x_{v_{j}}\right)=0$, and $\Delta^{\prime}\left(x_{v_{j}}\right)=0$ if $j \leqq m-1$, or

(c) $N_{n, k}$ is above (below) $M_{n, k}$ at $x_{v_{j-1}}$, and below (above) $M_{n, k}$ at $x_{v_{j}}$, according as $n-\nu_{j}$ is even (odd), and hence $\Delta(x)=0$ for some $x_{v_{j-1}}<x<x_{v_{j}}$.

In any case we recover one of the conditions (i) or (ii), with $j$ replaced by $j+1$. If we are in case (i) for $j=m$, the proof is complete. If we are in case (ii) for $j=m$, we may apply the induction argument once more, and again the proof is complete.

Lemma 6. If each of the $q$ disjoint closed intervals $K_{j} \subset K^{\prime}$, and if $\cup_{1}^{a} K_{j}$ contains $m$ of the points $x_{\nu}$, then $\Delta$ has at least $m-q$ zeros in $\bigcup_{1}^{q} K_{j}$.

Proof. This is clearly so for $q=2$, using Lemma 5 , and the result for arbitrary $q \leqq m$ follows by induction.

Returning now to the theorem proper, since $K^{\prime}$ contains at least $n+2 k+1$ $-n p+p^{\prime}-2 \sum_{1}^{p} \nu_{j}$ of the points $x_{\nu}$, and $K^{\prime}$ is the disjoint union of $p^{\prime}+1$ closed intervals, $\Delta$ has at least $n+2 k-n p-2 \sum_{1}^{p} \nu_{j}$ zeros in $K^{\prime}$ by Lemmas 5 and 6 , and hence at least $n+2 k-p^{\prime}-2 \sum_{1}^{p} \nu_{j}$ zeros in all. But we have already seen that $\Delta$ is a spline function of class $\left(n-1,2 k-1-p^{\prime}-2 \sum_{1}^{p} \nu_{j}\right)$, and thus it can have at most $n+2 k-1-p^{\prime}-2 \sum_{1}^{p} \nu_{j}$ zeros, by Theorem 4 . Thus $\Delta=0$, and the theorem is proved.

Finally, we must show that Theorem $2, n \geqq 2$, follows from Theorems 1 and 9 , the case $n=1$ of Theorem 2 having been settled in $\S 1$. Let $M_{n, k}$ be any monospline of class $(n, k)$ which has as its relative extrema the numbers $e_{\nu}=(-1)^{n-\nu}, 1 \leqq \nu \leqq n+2 k-1$, with $M_{n, k}\left(x_{\nu}\right)=e_{\nu}, x_{j}<x_{j+1}$. Such a monospline exists, by Theorem 1 . Let $x_{0}$ be the unique point to the left of $x_{1}$ such that $M_{n, k}\left(x_{0}\right)=(-1)^{n}$, and similarly let $x_{n+2 k}$ be the unique point to the right of $x_{n+2 k-1}$ such that $M_{n, k}\left(x_{n+2 k}\right)=1$. Such points surely exist, since $M_{n, k}$ is con- 
tinuous and monotone outside the interval $\left[x_{1}, x_{n+2 k-1}\right]$, by the remark following Theorem 6, and $M_{n, k}\left(x_{n+2 k-1}\right)=-1, M_{n, k}\left(x_{1}\right)=(-1)^{n-1}$. Let $2 \lambda=x_{n+2 k}$ $-x_{0}$, and $2 \mu=x_{n+2 k}+x_{0}$, and define the monospline $N$ of class $(n, k)$ by

$$
N(x)=\lambda^{-n} M_{n, k}(\lambda x+\mu) .
$$

Since $N$ has absolutely equal extrema at $n+2 k+1$ points of the interval $[-1,1]$, including both end-points, with alternating signs, $N=M_{n, \boldsymbol{k}}^{*}$ by Theorem 9, and the proof of Theorem 2 is complete.

4. The order of the approximation. Let $\delta_{n, k}$ denote the maximum value of $M_{n, \mathbf{k}}^{*}(x)$ on the interval $[-1,1]$. In the light of equations (8)-(11) it is not unreasonable to conjecture that $\delta_{n, k}=2^{1-n}\left(1+\lambda_{n} k\right)$, with $\lambda_{n} \rightarrow 0$ as $n \rightarrow \infty$. At any rate, it is of interest to bound $\delta_{n, k}$ above and below.

A presumably very crude lower bound may be obtained as follows. The $k$ knots of $M_{n, k}^{*}$ divide the interval $[-1,1]$ into $k+1$ subintervals, at least one of which must be of length $2(k+1)^{-1}$ or more. In this subinterval $M_{n, k}^{*}$ is a polynomial of degree $n$ with leading coefficient one, and hence cannot depart less from zero than does the polynomial $(1+k)^{-n} T_{n}[(k+1) x]$ in the interval $\left[-(1+k)^{-1},(1+k)^{-1}\right]$, where $T_{n}$ is as in (5). Hence

$$
\delta_{n, k} \geqq 2^{1-n}(1+k)^{-n} \text {. }
$$

As to an upper bound, the Bernoulli polynomials (see [1, chapter 5]) $B_{n}$ defined by

$$
\frac{z e^{x z}}{e^{z}-1}=\sum_{n=0}^{\infty} \frac{B_{n}(x)}{n !} z^{n}, \quad|z|<2 \pi, \quad 0 \leqq x \leqq 1,
$$

are known to satisfy the equation

$$
B_{n}(x+1)=B_{n}(x)+n x^{n-1} .
$$

From a consideration of the periodic extension of period unity of $B_{n}$ it is clear that the polynomial $B_{n}$, its leading coefficient being one, may be considered as a component of a monospline of class $(n, k)$. For $n$ odd, the functions $B_{n}$ are properly centered, i.e. $\max B_{n}(x)=-\min B_{n}(x)$. For $n$ even this is not the case, but it may be brought about by subtracting the quantity $2^{-n} B_{n}(0)$. Thus we consider instead of $B$ the function $B^{\#}$ defined by

$$
B_{n}^{f}(x)=B_{n}(x)-2^{-n} B_{n}(0),
$$

$B_{n}(0)$ being equal to zero for $n$ odd. Finally we change the variable so that the interval $[0,1]$ is mapped onto the interval $\left[0,2(1+k)^{-1}\right]$, defining

$$
B_{n, k}^{*}(x)=\frac{2^{n}}{(k+1)^{n}} B_{n}^{t}\left[\frac{1}{2}(k+1) x\right], \quad 0 \leqq x \leqq 2(k+1)^{-1} .
$$

Since $B_{n, k}^{*}$ is a component of a properly centered monospline of class $(n, k)$, 
$\delta_{n, k}$ is surely not greater than the maximum value of $B_{n, k}^{*}$ on the interval $\left[0,2(k+1)^{-1}\right]$. For $n$ even, this remark permits the estimate

$$
\delta_{n, k} \leqq \frac{\left|B_{n}(0)\right|}{(k+1)^{n}}\left(2^{n}-1\right) \leqq \frac{n !}{3 \pi^{n-2}(k+1)^{n}},
$$

while for $n$ odd we find similarly

$$
\delta_{n, k} \leqq \frac{2^{n-1} n\left|B_{n-1}(0)\right|}{\pi(k+1)^{n}} \leqq \frac{n !}{3 \pi^{n-2}(k+1)^{n}},
$$

so that the estimate

$$
\delta_{n, k} \leqq \frac{n !}{3 \pi^{n-2}(k+1)^{n}}
$$

is available in all cases. A comparison of (50) with (57) is disappointing in so far as the behavior of $\delta_{n, k}$ for fixed $k$ is concerned, as the coefficient of $(k+1)^{-n}$ tends strongly to zero in the first case and strongly to infinity in the second, as $n$ tends to infinity.

\section{REFERENCES}

1. C. Jordan, Calculus of finite differences, New York, Chelsea Publishing Co., 1947.

2. A. J. Kempner, On the shape of polynomial curves, The Tôhoku Math. J., Part I vol. 37 (1933) pp. 347-352; Part II vol. 42 (1936) pp. 318-330.

3. G. R. MacLane, Concerning the uniformization of certain Riemann surfaces allied to the inverse-cosine and inverse-gamma surfaces, Trans. Amer. Math. Soc. vol. 62 (1947) pp. 99-113.

4. Th. Motzkin, Approximation by curves of a unisolvent family, Bull. Amer. Math. Soc. vol. 55 (1949) pp. 789-793.

5. I. P. Natanson, Konstruktive Funktionentheorie, (German translation from Russian), Berlin, Akademie Verlag, 1955.

6. I. J. Schoenberg, On metric arcs of vanishing Menger curvature, Ann. of Math. vol. 41 (1940) pp. 718-722.

7. I. J. Schoenberg and Anne Whitney, On Pólya frequency functions. III. The positivity of translation determinants with an application to the interpolation problem by spline curves, Trans. Amer. Math. Soc. vol. 74 (1953) pp. 246-259.

8. I. J. Schoenberg, Spline functions, convex curves and mechanical quadrature, Bull. Amer. Math. Soc. vol. 64 (1958) pp. 352-357. 1942.

9. G. T. Whyburn, Analytic topology, Amer. Math. Soc. Colloquium Publications, vol. 38,

\section{University of Pennsylvania, Philadelphia, Pennsylvania \\ RAdIO CORPORATION OF AMERICA, MOORESTOWN, NEW JERSEY}

